\title{
SPACE-TIME DIVERSITY FOR MULTIMEDIA DELIVERY OVER WIRELESS CHANNELS
}

\author{
H. Zheng \\ Bell-Labs, Lucent T ec hnologies \\ 791 Holmdel-Keyport Rd., \\ Holmdel, NJ 07733, haitaoz@lucent.com
}

\author{
K.J.R Liu \\ Department of Electrical Engineering \\ University of Maryland,College Park, MD 20742 \\ kjrliu@eng.umd.edu
}

\begin{abstract}
A source-channel matched lay ered space-time diversity technique is presented for reliable delivery of lay er coded multimedia data through wireless channels. The transmission system is transformed to multiple subchannels and each associated with different div ersity gain, therefore different error performance. Similarly to the parallel transmission proposed for ADSL[9], we develop a parallel transmission scheme to match the source layers to the channel lay ers. $\mathrm{P}$ ow er con trol alters the error performance of each channel layer to ac hiev e the optimal performance.
\end{abstract}

\section{INTRODUCTION}

Dramatic development in the area of personal communications services allo ws wireless multimedia services. In the near future, it is expected that millions of users will have access to a wide variety of services that will be made av ailable over national and international communication netw orks. Mobile uses will be able to access their data and other services such as email, e-news including stock market news, video telephony, yellow pages, map services, electronic banking, etc. while on the move. Real-time multimedia services require high reliabilit $y$ with a low bounded time dela $y$ and a reasonably high transmission rate. Wireless channels on the other hand are error-prone, time-varying and band-limited. Automatic repeat request(ARQ) allows retransmission of the corrupted data, but yields long delay. Proper error control can be used to obtain more reliable transmission, and maintain a low delay. However, this causes unnecessary ov erhead and reduces throughput during periods of good channel status.

The derogatory effects of multipath fading in a wireless communication system can be mitigated by emplo ying artenna diversity. It inv olv es rultiple transmit an tennas and/or receive antennas to allow multiple signal replica at the receiver. Such advantage makes it to

This work $w$ as supported in part by NSF NYI Award MIP9457397. attractive to use for the transmission ov er band-limited wireless channels $[1,2,3]$. Recently, space-time diversity which achiev es both space and time diersity, has raised great interests $[4,5,6,7]$. Space-time codes which is a joint design of coding, modulation, transmit and receive diversity, has been developed for high data rate wireless communications[8].

The current diversity tec hniques are all developed for data transmission. In this paper, w econsider the problem of reliable multimedia data deliv ery through wireless channels using multiple transmit/receive antennas. Multimedia input can be represented in terms of a number of layers, eac $h$ resulting in a distinct data stream representing different perceptually relevan $t$ components of source material. Different layers may have distinctly different tolerances to channel errors and the corresponding data streams can then be handled differen tly b y the transmitter [ 9 In another word, the source lay ers receiv e unequal error protection(UEP). In [9], proposed a parallel transmission scheme for wireline channels, which transmits lay ers with higher error performance requirement through subchannels with higher channel gain to noise ratio. In this paper, we apply the parallel transmission scheme to lay ered space-time diversity technique.

\section{LAYERED SPA CE-TIME DIVERSITY}

We consider the layered space-time architecture proposed in $[5,6]$. It utilizes multiple antenna arra ys at both transmitter and receiver. The diagonal layered space-time (DLST) code distributes the coded data across diagonals in space-time domain. This structure leads to theoretical rates which increase linearly with the number of antennas at the transmitter and receiver. Horizonal layered space-time(HLST) code distributes the coded data among the rows in space domain, which leads to less complexity compared to DLST. By employing interference suppression and interference cancellation, the coded data layers can be separated and decoded independently. This results in a much lower decoding complexity compared to the trellis space-time 
codes which employ ML decoding, especially for large n umber of transmit an tennas. We consider HLST in the paper, where each antenna is considered as a channel la yer or subchannel.

The structure of HLST code is shown in Figure 1, assuming $N$ transmit an tennas and $M$ receive antennas. Let $\mathbf{t}=\left(t_{1}, t_{2}, \ldots, t_{N}\right)$ denote the vector of transmitted symbols and $\mathbf{r}=\left(r_{1}, r_{2}, \ldots, r_{M}\right)$ denote theeceiv ed signal,

$$
\mathbf{r}=H \mathbf{t}+\mathbf{v}
$$

where $\mathbf{v}$ represents the noisy vector and $H$ represents the channel gain matrix. Assuming perfect $H$ estimation at receiver, the decision variables from each horizontal location can be extracted and decoded. The receiv er first pic ks one layer, e.g. layer $N-1$, and extracts it by making a decision based on the received vector $\mathbf{r}$. Then the factor or contribution of layer $N-1$ to $\mathbf{r}$ is removed based on the decision of layer $N-1$. Therefore, lay er $N-2$ can be similarly decoded without the interference of layer $N-1$. Here we refer the layer as channellayer. For layer $k$, the probability of transmitting $\mathbf{t}$ and decoding $\mathbf{c}$ is[7]

$$
\begin{aligned}
P(\mathbf{t} \rightarrow \mathbf{c} \mid H) & =Q\left(\sqrt{\frac{E}{2 N_{0}} \sum_{l=0}^{N-1}\left|R_{l}^{k}\right|^{2}|\mathbf{t}-\mathbf{c}|^{2}}\right) \\
& \leq \exp \left(-\frac{E}{4 N_{0}} \sum_{l=0}^{N-1}\left|R_{l}^{k}\right|^{2}|\mathbf{t}-\mathbf{c}|^{2}\right) .(2)
\end{aligned}
$$

And the average error probability of layer $k$ can be approximated as

$\operatorname{Prob}(\mathbf{t} \rightarrow \mathbf{c}) \approx \prod_{\mathbf{c}, \mathbf{t}}\left(\left(|\mathbf{c}-\mathbf{t}|^{2}\right)^{-(M-k)}\right)\left(\frac{E}{N_{0}}\right)^{\sum_{\mathbf{c}, \mathbf{t}, \mathbf{c \neq} \mathbf{t}} M-k}$.

By applying Reed Solomon $(N, N-T+1)$ code, the symbol error probability for la yerk is then

$$
P e \approx\left(\frac{E d^{2}}{4 N_{0}}\right)^{-T(M-k)}
$$

where $d^{2}$ is the minimum distance between two neighboring modulation symbols. Therefore, lay er $k$ is associated with diversity gain $M-k$ and should have better error performance than lay er $N-1, . . k+1$ statistically.

\section{POWER CONTROL IN LAYERED SPA CE-TIME CODE}

HLST code is originally designed for data transmission. In suc h case, the design criteria is the error performance averaged over all the channel layers. Assuming each

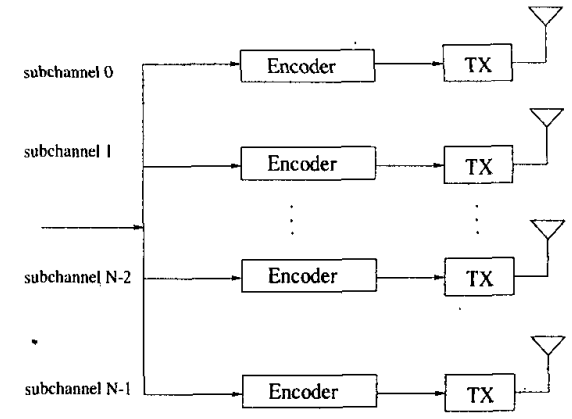

Figure 1: Horizontal Lay ered Space-Time Code

channel lay er uses the same transmitted pow er, the aerage SER of the channel lay ers can be computed as

$$
\begin{aligned}
\operatorname{Pe}(E) & =\frac{1}{N} \sum_{k=0}^{N-1}\left(\frac{E d^{2}}{4 N_{0}}\right)^{-T(M-k)} \\
& =\frac{1}{N}\left(\frac{E d^{2}}{4 N_{0}}\right)^{-T M} \frac{\left(\frac{E d^{2}}{4 N_{0}}\right)^{T N}-1}{\left(\frac{E d^{2}}{4 N_{0}}\right)^{T}-1}
\end{aligned}
$$

where the average power used $E_{T}=E$.

On the other hand, pow er allocation can also be applied to equalize the error performance of channel la yers. It leads to

$$
\begin{aligned}
& P e_{k}\left(E_{k}\right)=\left(\frac{E_{k} d^{2}}{4 N_{0}}\right)^{-T(M-k)}=\left(\frac{E_{l} d^{2}}{4 N_{0}}\right)^{-T(M-l)}=P e_{l}\left(E_{l}\right) \\
& \rightarrow E_{k}=\left(\frac{E_{0} d^{2}}{4 N_{0}}\right)^{\frac{M}{M-k}} \frac{4 N_{0}}{d^{2}} \text { and } \\
& P e_{k}=P e\left(E_{0}\right)=\left(\frac{E_{0} d^{2}}{4 N_{0}}\right)^{-T M},
\end{aligned}
$$

where $E_{0}$ represents the transmitted pow erfor channel layer $0 . \quad E_{0}$ is a function of $E_{T}=\frac{1}{N} \sum_{k=0}^{N-1} E_{k}$; therefore, the overall error performance can be represented as a function of $E_{T}$. How ever, due to the exponential factor $\frac{M}{M-k}$, such allocation leads to huge variation in the transmitted pow er assigned to the laers. Figure 4 illustrates the transmitted power for layer $N-1, \ldots, 0$ assuming $N=M=7$ and 8PSK modulation $d^{2}=0.7654$, as a function of $E_{T}$. As can be seen, the transmitted pow er assigned to lay er 6 increases dramatically as $E_{T}$ increases. This would yields difficulty in pow er amplifiers. Therefore, such approach is not feasible for practical applications.

\subsection{Optimization for Serial Transmission}

T otransmit layer coded multimedia data, the source layers can be transmitted consecutively as shown in 


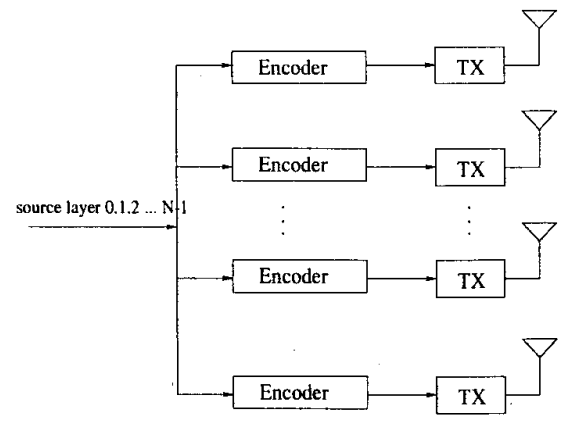

Figure 2: Serial Transmission in HLST

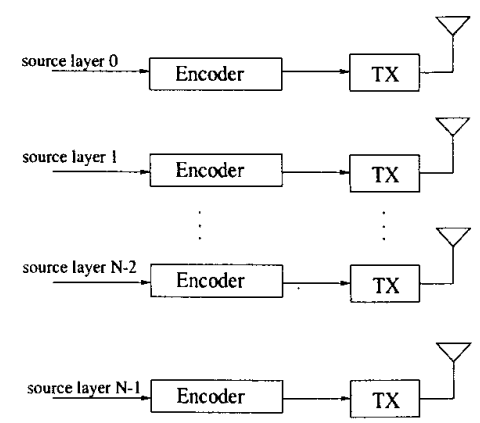

Figure 3: $\mathrm{P}$ arallel Tansmission in HLST

Figure 2, and the transmitted power can be assigned to minimize the approximated channel distortion

$$
\begin{array}{cc}
\min D_{c}=\sum_{l=0}^{N_{s}} W_{l} \operatorname{Pe}\left(E_{T}^{l}\right) \\
\text { subject to } & \sum_{l=0}^{N_{s}} S_{l} E_{T}^{l}=E_{b u d g e t},
\end{array}
$$

where $N_{s}$ is the n unber of source lay ers, $S_{l}$ is the symbol length of lay er $l$. Similar to previous sections. LaGrange multiplier method can be applied to find the optimal solution.

\subsection{Optimization for Parallel Transmission}

F or HLST code, hannel lay ers are associated with differen terror performance. Rather than equalizing or av eraging the error performance among all the channel lay ers, w epropose to transmit different source layers as different channel lay ers, as sho wnin Figure 3. We assume $N=N_{s}$ so that each source lay er is mapped to one $c$ hannel lyers. The combined source-channel layer approach can allocate the transmitted pow er andthe error control coding rate to achieve the optimal error performance distribution. The distortion minimization function can be formulated as

$$
\begin{aligned}
\min & \sum_{k=0}^{N-1} W_{k}\left(\frac{E_{k} d^{2}}{4 N_{0}}\right)^{-T_{k}(M-k)} \\
\text { subject to } & \sum_{k=0}^{N-1} S_{k} E_{k}=E_{\text {budget }} .
\end{aligned}
$$

Here w eassume that $S_{k}=S, k=0 \ldots N-1$. Using LaGrange multiplier, the optimal pow er distribution satisfies

$$
E_{k}=\frac{4 N_{0}}{d^{2}}\left(\frac{W_{k} T(M-k)}{S \lambda}\right)^{\frac{1}{T(M-k)+1}}
$$

and $\lambda$ satisfies

$$
\sum_{k=0}^{N-1} S \frac{4 N_{0}}{d^{2}}\left(\frac{W_{k} T(M-k)}{S \lambda}\right)^{\frac{1}{T(M-k)+1}}=E_{\text {budget }} .
$$

Since the left hand side is a monotonic function of $\lambda$, the optimal solution can be computed using Newton or bisection methods.

\section{SIMULATION RESULT}

The transmission system employs 7 transmit antennas and 7 receive antennas. We apply Reed Solomon $(7,3)$ code with 8PSK modulation. Therefore, each diagonal con tains 7 8PSK symbols or a RS block. The corresponding parameters are $d^{2}=0.54$ and $T=7-3+1=$ 5. For simplicity, w eassume the source multimedia data are decomposed into 7 equal length source layers, eac $\mathrm{h}$ associated with a weighting factor $W_{k}, k=$ $0, \ldots, N-1$. We compare the serial transmission and parallel transmission system in terms of the mean square error of the transmitted and received data, as shown in Figure 5. For serial transmission, equal power distribution in (5) is achieved. P arallel approach outperforms serial approach in low SNR region. As SNR increases, the performances converge. The actual symbol error rate of source layers are also illustrated in Figure 6 and 7 . The parallel approach utilizes the property of HLST code and achieves UEP efficiently. The SER performance for the most important source lay ers causes the performance bottleneck for serial transmission, since huge amount of transmitted pow er has to be assigned to reduce the noise effect in the channel layer $N-1, N-2 \ldots$.

\section{CONCLUSION}

By matching the source layers to channel lay ers of Layered space-time codes, we propose a parallel transmission sc heme utilizing the natural UEP in HLST armitecture. $\mathrm{P}$ ow er allocation is performed to optimize the 
error performance according to the importance of the source lay ers.

How eer, the space-time codes rely on being able to accurately estimate the fading coefficients which are assumed to vary slowly. Therefore, the need of powerful and yet efficient channel estimation or equalization techniques is very important. In this paper, w e assume perfect channel estimation. F uture work may incorporate the channel estimation into the system and investigating the o erall performance.

\section{REFERENCES}

[1] G. W. Wornell and M.D. Troot, "Efficient Signal Processing Techniques for Exploiting Transmit Antenna Diversity on $\mathrm{F}$ ading Channels", IEEE T rans. on Signal Processing, pp. 191-205, Jan. 1997.

[2] J.H.Winters, "The Diversity Gain of Transmit Diversity in Wireless Systems with Rayleigh Fading", Proc. of ICC'94, pp.1121-1125, June 1994.

[3] G. J. Foschini and M. J. Gans, "On Limits of Wireless Communications in a $F$ ading En vironment When Using Multiple Antennas", Wireless Personal Communications, pp. 311, Vol. 6, No. 3, March 1998.

[4] G.Rayleigh and J.M. Cioffi, "Spatial-temporal Coding for Wireless Communications", Proc. IEEE Globecom96,pp.1809-1814.

[5] G. J. Foschini, "La yeredSpace-Time Architecture for Wireless Communication in a Fading En vironmet When Using Multiple Antennas", Bell Labs T echnical Journal, pp 41-59, Vol. 1, No. 2, Autumn 1996.

[6] G. D. Golden, G. J. Foschini, R. A. Valenzuela, P. W. Wolniansky, "Detection Algorithm and Initial Laboratory Results using the V-BLAST Space-Time Communication Architecture", Electronics Letters, Vol. 35, No. 1, Jan. 1999, pp. 14-15.

[7] D. Shiu and J.M. Kahn, "Design of High-Throughput Codes for Multiple-Antenna Wireless Systems", submitted to IEEE Trans. on Information Theory, Jan. 1999.

[8] V. Tarokh, N. Seshadri and A.R. Calderbank, "SpaceTime Codes for High Data Rate Wirèless Communication:Performance analysis and code construction", IEEE Trans. on Information Theory, pp.744-765, vol.44, No.2, March 1998.

[9] H. Zheng and K.J.R. Liu, "Robust Image and Video T ransmission over Spectrally Shaped Channels Using Multicarrier Modulation", IEEE Trans. on Multimedia, pp. 88-103, Vol.1, No.1 March, 1999.

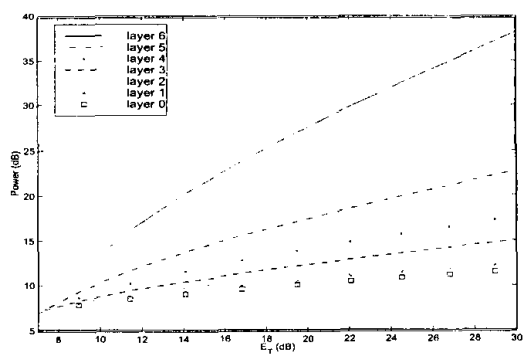

Figure 4: La yer $\mathrm{P}$ ow er Distribution

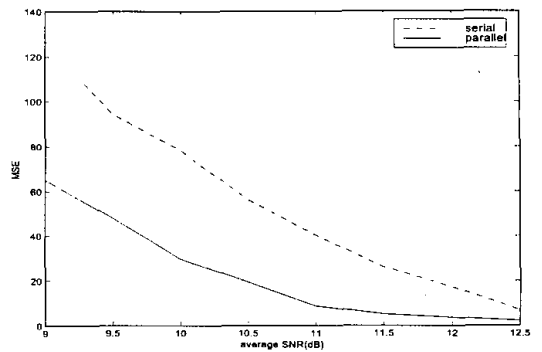

Figure 5: MSE Performance Comparison of serial and $\mathrm{P}$ arallel Tansmission

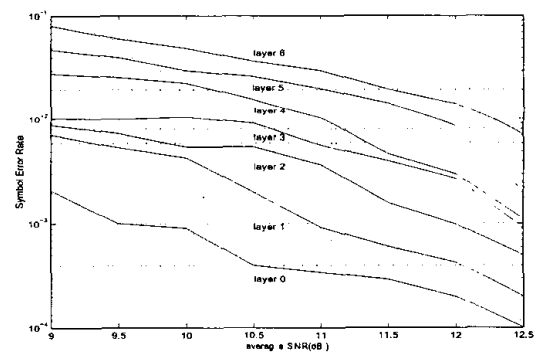

Figure 6: Serial T ransmission: La y erSymbol Error Rate Distribution

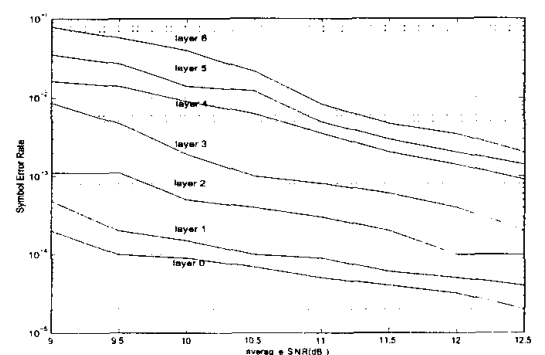

Figure 7: $\mathrm{P}$ arallel T ransmission:Lyer Symbol Error Rate Distribution 